improved; of those from thirteen to eighteen years of age, almost two thirds were straightened, one third markedly improved; of those from nineteen to twenty-five, almost one half were straightened, one half were markedly improved; of those from twenty-six to thirty-five, over one half were straightened, one half were markedly improved. In other words, much may be expected with much deformity at all ages where there is a low degree of rigidity.

Of the more rigid cases, under twelve years of age, one half were greatly improved, one fifth were markedly improved; of those from thirteen to eighteen years of age, one fifth were greatly improved, one half markedly improved; of those from nineteen to twenty-five years of age, one fourth were greatly improved, three fifths were markedly improved.

Of the extremely rigid cases, under twelve years of age, one fourth were markedly improved; of those from thirteen to eighteen, three fifths were markedly improved; of those from nineteen to twenty-five, one third were markedly improved, and of those from twenty-six to thirty-five, one third were markedly improved.

The amount of rigidity, while not absolutely a guide to the ultimate outcome, the improvement is largely proportionate to it.

The length of treatment averaged two and one-third years in cases that were greatly improved and cases that were markedly improved. Of the cases that were not improved, only one was treated any length of time; that was a hopeless case from the start. Of the cases that received no benefit, 10 were treated one year, 9 were very irram' $r$.

s not as important a factor as would be $\mathrm{d}$, but it is an important factor, as may we seen below; one half of the cases that were greatly corrected were under eighteen; one third were over twenty-five. Cases that were markedly improved, over one half were under eighteen, one third were over twenty-five.

The general health, muscular condition and endurance is greatly benefited in all cases. To make an impression on the curve, length and perseverence in treatment is more of a factor than age, but the amount of rigidity of the curve is more important than the amount of curve. Age is a factor, but two thirds of those over twentyfive were greatly corrected or greatly benefited. Almost all cases may be improved; some greatly improved and severe cases with moderate rigidity may be greatly corrected.

While in these stiff and extremely deformed cases the treatment requires a good deal more time than for simple curves, and for some cases there is a sacrifice of personal comfort for a short period, still the fact remains that it is effective treatment and one of the easiest, and for the patient a most comfortable way to correct difficult rigid structural scoliosis in a comparatively short time. The future of the patient, the health, and to prevent a crippled or semi-invalid condition makes it necessary not to trifle in the treatment of advanced cases of scoliosis. The slight curves may be treated with much less sacrifice of time and less strenuous work, depending on the severity of the case. Difficult cases of scoliosis, with complicated curves, rotation and rigidity form a class which are often very discouraging to treat. The writer wishes to make a plea for very rigorous treatment in these cases, for not only may they be vastly improved, but treatment can in most cases prevent them from being badly deformed or crippled later on.

\section{ACUTE DILATATION OF THE STOMACH FOL-} LOWING SURGICAL OPERATIONS.*

BY EDWARD WALLACE LEE, M.D., NEW YORK.

THIS is an infrequent complication, but one of the most serious that may follow any surgical procedure. It is more liable to follow operations in the abdominal or pelvic cavities. Laymen frequently facetiously remark: "Doctor, you may consider your operation successful, but your patient died." This remark is often true. The operation has been successful, but an unforeseen, unavoidable, and often fatal complication, especially of this character, brings about unjust and unfavorable criticism of the surgeon's work. My limited experience has demonstrated the seriousness of this complication, and the subject is presented to this Association more for discussion than for any definite information the writer may have to impart.

It is fitting that this subject should be discussed before a society of this character, made up, as it is, of general practitioners and the exponents of many of the different so-called specialties in medicine. Frequently the surgeon after operation leaves his patient under the care of the family doctor, and, therefore, the latter must assume the responsibility of the after-treatment. He should be well prepared to recognize the various complications which may arise, and as this is one of the most serious, it ought not to be overlooked. As has been stated, it is especially liable to follow operations in the abdominal or pelvic cavities. We often see after abdominal section various gastric and intestinal disturbances which greatly complicate and retard recovery, and the early recognition of these may sometimes prevent a fatal termination.

\section{ETIOLOGY.}

My own observations have not been such as to enable me to assign a cause that would directly produce this condition. There are many theories as to its cause,- the anesthetic, extent of operation, its location, all have been given as possible causes, but experience so far has shown that this sequela may follow various surgical procedures, regardless of the anesthetic, location, extent or character of the operation. Various other causes have been mentioned, such as spasm of the pylorus, volvulus of the stomach, kink in the duodenum, and pressure on the mesenteric vessels (irritation of the solar plexus, after removal of large uterine fibroid tumors, permitting of general ptosis of the

* Presented at the meeting of the Medical Association of the Greater City of New York, Feb. 20, 1911. 
abdominal contents, which press down on the mesenteric vessels, thereby interfering with proper circulation and nerve function). But it is not to mechanical causes that I especially refer, for of course if existing, they are self evident.

It is a question whether this condition is due to a neurosis producing paralysis permitting the formation of gases, which would naturally be followed by gastric dilatation, or to an irritation continuously producing excessive gastric secretion. From my observation I am inclined to look upon it as due to a neurosis, some serious vasomotor nerve disturbance, rather than to any direct irritation of the stomach itself. Even after the stomach has been thoroughly irrigated, having been emptied of its contents, it will rapidly refill with secretion and become enormously distended with gas, and on post-mortem examination it has been found that the dilated stomach was free from contents with the exception of gas.

\section{DIAGNOSIS AND CHARACTERISTICS.}

There are certain signs and symptoms of acute gastric dilatation which may be considered pathognomonic. On physical examination we find the stomach dilated sometimes to its normal capacity, but often extending into the pelvis. The condition is diagnosed by inspection and palpation, and the various lines of symptoms which accompany it. It usually comes on anywhere from one hour to twelve to fourteen hours after the operation. In one case I observed it within an hour after the patient had been placed in bed. The earlier symptoms are apparently those of shock, and when I say shock, I mean that extreme shock in which intolerable vomiting occurs. The contents of the stomach are followed by expulsions of glairy mucus, and, if the vomiting continues, of mucus streaked with blood. Often the vomitus is brought up in large gulps without straining. Aside from the vomiting, the surgeon's attention should be attracted by the rapid and constantly increasing pulse. The symptoms are often allied to those of internal hemorrhage, but this complication can usually be excluded by taking into consideration all the details of the operation.

The general condition of the patient is recognized as being serious from the first. The respirations are frequent and shallow, the pulse rapid, thready and of poor quality, and the skin often cold and clammy. The aspect of the patient indicates that his tissues are deficient in water; he is pinched and careworn, and appears to have aged suddenly. He is apt to be restless, and at times irritable. Diarrhea has been observed, and the urine is nearly always scanty. On palpation and inspection the stomach is usually found enormously distended and sharply bent upon itself, so as to form a gigantic " U." Generally on the lesser curvature there is a sharp kink. The stomach occupies the greater part of the abdominal cavity, cutting off the large and small intestines. Of course, the stomach walls are tightly stretched and consequently thin.

From an anatomical point of view there is a characteristic condition which is self-evident. That the stomach is enormously distended can be easily recognized, especially in thin patients, by the distention extending toward the left side; even causing bulging of the ribs as it forces itself up under these and presses on the diaphragm, thereby causing mechanical interference with the heart's action. This mechanical interference, combined with the general systemic shock, brings about a condition which it can be readily understood, is likely to prove fatal. I emphasize the distention toward the left because on the right side of the upper abdominal region there is flatness on percussion, the abdominal wall at times being retracted and allowing the liver to be easily palpated. This point is worthy of special attention, as it is the means, if recognized, of greatly aiding in making a diagnosis of acute gastric dilatation.

I have seen a number of these cases, but will report but four or five. The cases observed have been males (between the ages of forty-three and seventy) with one exception, and this exception will be referred to later on.

CASE I. A man forty-three years old had been operated for appendicitis. Two years later he developed hernia on the side of the operation. He was operated on for the hernia. He was a strong, robust man, with apparently no gastric or intestinal disturbances, but, being an army officer, it became necessary that his hernia should be operated on. Within four hours after the operation there was an enormous distention of the stomach - gastric, not intestinal, dilatation. The line of treatment which will be described was followed out. This case made a recovery.

The following three cases terminated fatally.

CASE II. Man, sixty-five; gall-bladder case; removal of large single stone and the gall bladder. Within eight hours after the operation there developed an acute dilatation of the stomach. The general line of treatment was followed out, but death ensued thirtysix hours after the operation.

CASE III. Suprapubic prostatectomy; a man sixty years old. Acute dilatation of the stomach followed within fourteen hours after the operation. In this case the patient was a cocaine habitué of highly nervous temperament and physically in bad condition. What these elements had to do with bringing about the acute dilatation and fatal termination are questions for your consideration.

Case IV. Suprapubic cystotomy for chronic purulent cystitis. Within one hour after the operation acute dilatation of the stomach with all its characteristic and distressing symptoms was apparent. Every form of treatment indicated was carried out. Fatal termination forty-eight hours after the operation.

As I have said, the cases I have observed have been males with one exception. This was a woman twenty-six years old, and the case does not, strictly speaking, belong to this category, as the acute dilatation did not follow surgical operation, but a burn. The latter was of the second degree, in extent about six inches square, and situated on the upper abdomen. The burn in itself was not serious enough to have produced the symptoms which followed within three hours after the injury, but within that period there was acute dilatation 
of the stomach with all its characteristic symptoms. The case terminated fatally twelve hours after the injury. I refer to this case as tending to carry out the idea that acute dilatation of the stomach is of nervous origin rather than due to actual mechanical irritation of the stomach itself.

\section{TREATMENT}

As has been stated, we find the patient in profound shock and the stomach enormously distended, not only with secretion, its so-called normal contents, but gas. The indication is to resort as soon as possible to remedies which will th relieve shock and sustain the patient. Mechanical irrigation, washing out of the stomach, is primarily indicated. The stomach should be relieved of its other contents and gas by the introduction of the stomach tube, and if necessary the tube should be left in until there is no further indication for its use. In some cases it has been observed that even after thorough evacuation of the stomach contents, and the liberation of the existing gas, the stomach will rapidly refill not only with secretion, but with gas; therefore in many instances the permanent use of the tube is indicated.

The drugs indicated are, first, morphine, to relieve pain and shock, and next, strychnine, to stimulate the heart's action. This is given also for its effect on the vasomotor nervous system, with the hope that it may induce contraction of the distended stomach, for we know that strychnine is a powerful contractor, if I may use the term, stimulating the contraction of the involuntary muscles. We know that it is of service in acute dilatation of the heart, and we know that it is one of the most reliable remedies in post-partum uterine dilatation. Another remedy in use is eserine, which is indicated on account of its efficiency in bringing about peristalsis. This drug is strongly advised if there is any evidence that the colon or the small intestines are implicated in the existing distention.

Various surgical procedures have been recommended, none of which I have ever resorted to, for in the first place, the shock is so severe that any operation would necessarily increase the th amount of existing shock. Gastrostomy and gastro-enterostomy have been suggested. The latter, I believe, is too complicated a procedure to be even thought of. Gastrostomy could be resorted to under local anesthesia, and I believe that it has certain indications which are at least theoretically logical.

The general line of treatment resorted to after all surgical procedures should, of course, be carried out to bring about if possible a favorable termination, though this condition is of so serious a character that a majority of the cases $(75 \%)$ terminate fatally. So grave an affection should receive our most earnest consideration, and this, therefore, is the principal reason why the subject has been presented to you. The general literature and general discussion of this subject have been too limited for a condition so serious and important.

\section{ACUTE POST-OPERATIVE DILATATION OF THE STOMACH.*}

BY ROBERT T. MORRIS, M.D.,

Professor of Surgery at the New York Post-Graduate Medical School and Hospital.

WHEN an artist cannot draw very well, he turns to making impressionist views, and that is the sort of picture I would draw of this condition. Acute post-operative dilatation of the stomach presents four salient features: (1) The initial disturbance; (2) increased peristalsis and antiperistalsis; (3) great dilatation of the stomach walls; (4) extreme amount of secretion.

We can group a number of features in the perspective.

(a) Hormones secreted in the stomach mucosa stimulate activity of secretory glands.

(b) Chromaffin secretions from ductless glands and sympathetic ganglia control motor activity in part, and conduct trophic influence in part.

(c) The cerebrospinal nerves conduct motor activity.

Shock, ether and toxins are all known to derange the action of chromaffin secretions, and that could account for the deranged action of the sympathetic nerves in these cases. Shock, ether and toxins may overstimulate the sympathetic nerves. Overstimulation of the inhibitory nerves of the stomach would have about the same result as cutting the vagus, which we know experimentally leads to enormous dilatation of the stomach. The stomach, like the heart, has a systole and diastole. So much for the dilatation part of the picture.

Shock, ether and toxins may all derange the action of the hormones, with consequent loss of control of the secretory glands, which would then give us some products in excessive proportion, and other products in diminished proportion. So much for the vast quantities of fluid in the stomach in these cases, and for the gas, when saprophytes take charge of material given over to them by deranged digestion controllers.

Shock, ether and toxins may all disturb the cerebrospinal system, but we may assume that this later development of the embryo is more resistant and less easily influenced than the primitive sympathetic system. This idea places the cerebrospinal system practically out of our picture.

Now let us get theories into concentrated space, and use Dr. Lee's case of burn of the abdomen for a bull's-eye. Let us think at a target, and see if we can hit that bull's-eye. It was a small burn of the upper abdomen, yet the patient had acute dilatation of the stomach and died in two days. How could a small superficial burn do all that?

Heat transforms some albuminous tissue into

* Presented at the meeting of the Medical Association of the Greater

At the meeting of the Medical Association of the Greater City of New York, on Feb. 20, Dr. Edward Wallace Lee read a paper on the subject of "Acute Dilatation of the Stomach (Journal, page 562). He reported upon cases of his own, and among these was a case following a small burn of the abdomen. Our regular correspondent, who was present, has suggested that we publish the discussion of Dr. Morris in full, and as an original contribution. 